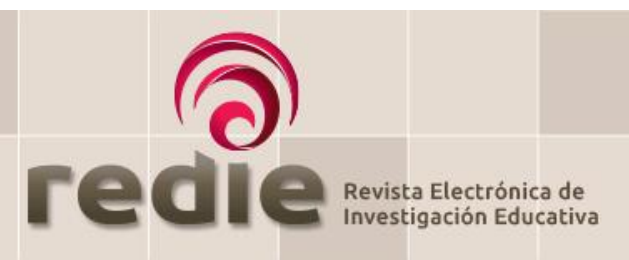

Vol. 21, 2019/e01

\title{
Impacto de las Transferencias Monetarias Condicionadas en la profesión académica en México: distintos tiempos, diferentes condiciones
}

\section{Impact of Conditional Monetary Transfers on the Academic Profession in Mexico: Different Times, Different Conditions}

\author{
Manuel Gil-Antón (*) mgil@colmex.mx \\ Leobardo Eduardo Contreras Gómez (*) lcontreras@cinvestav.mx \\ (*) El Colegio de México \\ (Recibido: 12 de mayo de 2018; Aceptado para su publicación: 26 de junio de 2018)
}

Cómo citar: Gil-Antón, M. y Contreras, L. E. (2019). Impacto de las transferencias monetarias condicionadas en la profesión académica en México: distintos tiempos, diferentes condiciones. Revista Electrónica de Investigación Educativa, 21, e01, 1-15. doi:10.24320/redie.2019.21.e01.2443

\section{Resumen}

Este trabajo analiza la profesión académica en México en dos períodos: cuando la conducción de las trayectorias académicas era regulada por la vía salarial y, posteriormente, cuando se orientaron por el "pago por mérito". El análisis emplea los datos de la investigación "La reconfiguración de la profesión académica en México" (2007) para los integrantes del Sistema Nacional de Investigadores, y divide la muestra en dos grupos: veteranos y jóvenes. Los primeros se ubican en la primera época y los segundos en la época en que las carreras académicas ya eran guiadas por las Transferencias Monetarias Condicionadas. Se construyeron tres indicadores: el lapso transcurrido para la obtención del doctorado, el tiempo que fue necesario para lograr el primer trabajo de tiempo completo y las condiciones formativas para el ingreso a la carrera académica. Los resultados de la comparación muestran que las Transferencias Monetarias Condicionadas modificaron los tiempos de formación e incorporación a la planta académica, así como los niveles formativos para iniciar las trayectorias.

Palabras clave: Enseñanza superior, política educacional, personal docente, cambio organizacional.

\section{Abstract}

This study explores the academic profession in Mexico at two times: when academic careers were governed by salaries, and the subsequent period in which they were regulated by "merit-based pay". This analysis uses data from the study "The Changing Academic Profession (CAP) in Mexico" (2007) with respect to members of the National System of Researchers (SNI), and splits the sample into two groups: veterans and young academics, the former from the first period and the latter from the period when academic careers were shaped by Conditional Monetary Transfers (TMC, in Spanish). Three indicators were constructed: time taken to obtain a doctorate degree, time taken to hold a full-time position, and 
previous education upon entry into an academic career. The results of the comparison show that Conditional Monetary Transfers changed the timing of education and entry into the academic career, and the level of education of researchers beginning their career.

Keywords: Higher education, educational policy, higher education teaching personnel, organizational change.

\section{Introducción}

Humanos al fin, estamos siempre en el río del tiempo. ¿Qué es el tiempo? Rolando García, en un seminario dedicado a la epistemología constructivista, aportó una manera de concebirlo: no sabremos nunca, expresó, si el tiempo existe con independencia de nosotros. ¿Cómo, entonces, delimitar a qué nos referimos con ese término? Su propuesta fue: "El tiempo es un parámetro de la transformación de la materia" (García, 1998). Las escalas temporales y su duración las proponemos para aproximarnos a una manera de dar cuenta del cambio en los fenómenos físicos.

De manera análoga, en la Historia y en las Ciencias Sociales, el tiempo es un "parámetro" de la transformación de las condiciones de contorno en que se establecen, de manera variable, tipos de relaciones, instituciones y estructuras dominantes que orientan y dan sentido a las trayectorias y acciones de los sujetos que, en ese lapso y coordenadas espaciales específicas, viven y desempeñan distintos roles.

Es propio de nuestra especie -hasta donde es posible saberlo- establecer períodos que se diferencian por ciertas características o condiciones que ocurren y advertimos contrastantes por sus efectos en las relaciones sociales. En la historia de la educación superior en México, y la profesión académica que le es convergente, hay varios modos posibles de periodizar, que están al servicio de diferentes preguntas con respecto al cambio, a la transformación.

¿Hay evidencia de modificaciones en los tipos de trayecto predominantes en el contexto del cambio de regulación salarial, hasta mediados de los años ochenta del siglo XX, al pago por mérito, iniciado en 1984, en el espacio laboral, especializado en la investigación académica? Lo que se propone en este artículo es que, en efecto, hay un antes y un después de la puesta en práctica de las Transferencias Monetarias Condicionadas (TMC), vistas como indicador de un cambio en las condiciones sociales de contexto más complejo y, por ende, multifactorial. Estos cambios se aprecian en tres grandes dimensiones: la duración de los procesos formativos, las condiciones y lapsos en cuanto al ingreso a un empleo de tiempo completo en el espacio laboral académico y, por ello, el nivel de estudios necesario para iniciar la carrera académica.

\subsection{Períodos}

Existe una primera modalidad en la generación de este espacio laboral al interior de la educación superior pública en México: se trata de la etapa salarial. Aproximadamente hasta 1989, la carrera académica era reconocida por tabuladores y contratos, o condiciones generales de trabajo, que se reflejaban en categorías y niveles, con mayores ingresos en correspondencia con la complejidad de las funciones a desempeñar (Gil-Antón, 2014).

Derivado de la crisis en la década de los ochenta, que implicó una caída sustantiva de la capacidad del poder adquisitivo de los ingresos, surge, paulatinamente, un segundo período en el que la resolución de la merma de los salarios ocurre a través de lo que se conoce como pago por mérito (Galaz y Viloria, 2014; Galaz et al., 2012; Ordorika, 2004; Ordorika y Lloyd, 2014) que consiste en otorgar recursos adicionales a quienes, de manera individual, tengan o adquieran ciertas condiciones formativas y produzcan lo que se espera de un académico con perfil deseable.

De esta manera, las autoridades educativas del nivel superior, en los años noventa, enfrentaron dos problemas: la recuperación del ingreso, sí, pero de manera individualizada (ya no salarial-contractual) y, a través de las condiciones establecidas para ello, dar paso a la "reingeniería" de la planta académica contratada aceleradamente en el período de expansión, junto con el establecimiento de nuevas 
condiciones para el ingreso a este espacio laboral.

Esta investigación tiene como principal referente el trabajo de Galaz y Viloria (2014), en el cual se analizan las variaciones más relevantes que implicó para la profesión académica el paso de una etapa a otra. A diferencia de ese estudio, el que ahora se presenta está centrado en investigadores adscritos al Sistema Nacional de Investigadores (SNI). Hay confluencia en los hallazgos de ambos trabajos, pero el hecho de realizarlo en la "élite" de la profesión académica, y comparando áreas del conocimiento, ahonda la pertinencia de la conjetura que orienta a las dos indagaciones.

Uno de los instrumentos de este cambio de época es el SNI, que coincide con las características ya referidas de las TMC. No se afirma que el sNI fuese "el cambio" en la lógica de esta nueva etapa, sino que es una consecuencia, entre otras, de la transformación de las condiciones para el desarrollo de la carrera académica (López-Olmedo, Marmolejo-Leyva, Pérez-Angon y Zayago-Lau, 2017; Payró, García y Aquino, 2016; Rodríguez, González-Brambila y Maqueda, 2018).

Sobre este programa se han realizado diversos estudios (Flores, 2014; Malo, 2005; Rodríguez et al., 2018) que muestran el impacto y las consecuencias de su instauración. Sin embargo, lo que guía este trabajo es estudiar, comparativamente, las modalidades típicas de ciertos tramos de las trayectorias entre los académicos de ambas etapas, contrastando las características tanto de sus procesos formativos como de la incorporación al mercado laboral académico (Ordorika, 2004) en las distintas condiciones de contorno socioeducativas propias del "antes y el después" de la preeminencia de las TMC.

Hay un sistema de reglas para el desempeño en las tareas académicas previas a la introducción de las TMC en la orientación de las carreras académicas, que es diferente a cuando se establecieron y se consideran parte de un orden establecido y natural en el campo, fuente no sólo de ingresos adicionales, sino de otros incentivos, como el prestigio y la distinción. ${ }^{1}$

Se ha establecido un acuerdo general en torno a la relevancia de esta lógica procedimental en la orientación de las trayectorias académicas de los investigadores (López-Olmedo et al., 2017). Sin embargo, no fue percibido así en el momento de su fundación, ya que incluso se pensaba como un programa temporal (Diario Oficial de la Federación,1984; Flores, 2014; Sarukhán, 2005; Sarukhán, 2014). No fue así, continúa luego de 34 años.

\section{Método}

En el contexto de la investigación "La Reconfiguración de la Profesión Académica en México" (RPAM), que fue parte de la indagación comparativa internacional "The Changing Academic Profession" (CAP), en 2007 se aplicó un cuestionario a una muestra nacional y, adicionalmente, a un grupo amplio de académicos del SNI (Galaz et al, 2012; Red de Investigadores sobre Académicos [RDISA], 2009). Mientras la muestra nacional se construyó intencionalmente con todos los requisitos para poder ser representativa del personal académico de tiempo completo del país en ese año, la aplicación del mismo cuestionario a los académicos del SNI (RDISA, 2007) fue por medio de una invitación a todos sus integrantes para que respondieran por vía electrónica. Esto significó no poder asegurar aleatoriedad ni representatividad por niveles en el sistema, áreas del conocimiento y otros valores de la población de la que formaban parte. ${ }^{2}$

Por lo tanto, para proponer conjeturas empíricas con cierto grado de verosimilitud en relación con los integrantes del SNI, una alternativa es comparar las distribuciones de ciertos valores relevantes de la muestra, con los correspondientes del total de integrantes del sistema para el mismo año.

\footnotetext{
${ }^{1}$ Es cierto que se establecieron, por primera vez, con el sNI, pero años después, con modalidades diversas, se implantaron en cada una de las universidades públicas del país, y otros sitios en que se lleva a cabo la producción de conocimientos.

${ }^{2}$ Se trabajó con la información de 2007, pues es la más reciente que existe en la que constan las variables necesarias para la investigación. Está en curso, aunque en etapas iniciales, otra investigación que permitirá actualizar los datos.
} 
De 13,485 académicos adscritos al SNI en el 2007 (Rodríguez, 2016), 5,339 contestaron completo el cuestionario (39.6\%). Para poder emplear la muestra como base de proposiciones razonables es necesario que su distribución sea semejante a la manera en que se distribuían, en ese entonces, el total de integrantes del sistema por áreas del conocimiento y entre los niveles de pertenencia que contiene. Se obtuvieron los datos para todos los integrantes del SNI de los años 2007 y 2008 (Rodríguez, 2016), de tal manera que se pudiesen cotejar. En la tabla I se da cuenta de la comparación entre la muestra y el total de los investigadores del sistema para esos años desglosando áreas del conocimiento, y en la tabla II se presenta el desagregado de los investigadores por su nivel dentro del sNI.

Tabla I. Distribución de académicos RPAM 2007/SNI por área del conocimiento y distribución en el conjunto del sNI

\begin{tabular}{l|l|c|c|c|c|c|c|c|c}
\hline \multicolumn{1}{l}{} \\
\cline { 3 - 10 } \multicolumn{1}{l}{\begin{tabular}{l} 
Muestra \\
\cline { 3 - 10 } Cuestionario
\end{tabular}} & 1 & 2 & 3 & 4 & 5 & 6 & 7 & Aotal \\
RPAM/SNI 2007 & Académicos & 834 & 835 & 386 & 785 & 747 & 604 & 762 & 4953 \\
\hline \multirow{2}{*}{ SNI 2007 } & Porcentaje & 16.9 & 16.9 & 7.8 & 15.8 & 15 & 12.2 & 15.4 & 100 \\
\hline \multirow{2}{*}{ SNI 2008} & Académicos & 2277 & 2179 & 1429 & 2169 & 1854 & 1587 & 1990 & 13485 \\
& Porcentaje & 16.9 & 16.2 & 10.6 & 16.1 & 13.7 & 11.8 & 14.8 & 100 \\
\hline
\end{tabular}

Nota 1. Area1: Ciencias Físico-Matemáticas y Ciencias de la Tierra. Área 2: Biología, Química y Ciencias de la Vida. Área 3: Medicina y Ciencias de la Salud. Área 4: Humanidades y Ciencias de la Conducta. Área 5: Ciencias Sociales. Área 6: Biotecnología y Ciencias Agropecuarias; Area 7: Ingenierías.

Nota 2. Elaboración propia con datos de Rodríguez (2016).

Tabla II. Distribución de académicos RPAM/SNI por nivel de adscripción y composición por nivel del conjunto del sNı

\begin{tabular}{|c|c|c|c|c|c|c|}
\hline \multirow{2}{*}{\multicolumn{2}{|c|}{ Muestra }} & \multicolumn{5}{|c|}{ Nivel } \\
\hline & & \multirow{2}{*}{$\begin{array}{c}\text { Candidato } \\
1002\end{array}$} & \multirow{2}{*}{$\begin{array}{c}1 \\
2918\end{array}$} & \multirow{2}{*}{$\begin{array}{c}2 \\
803\end{array}$} & \multirow{2}{*}{$\begin{array}{c}3 \\
250\end{array}$} & \multirow{2}{*}{$\begin{array}{l}\text { Total } \\
4973 \\
\end{array}$} \\
\hline Cuestionario & Académicos & & & & & \\
\hline RPAM/SNI 2007 & Porcentaje & 20.1 & 58.7 & 16.1 & 5.0 & 100 \\
\hline \multirow{2}{*}{ SNI 2007} & Académicos & 2389 & 7565 & 2428 & 1103 & 13485 \\
\hline & Porcentaje & 17.7 & 56.1 & 18.0 & 8.2 & 100 \\
\hline \multirow{2}{*}{ SNI 2008} & Académicos & 2589 & 8165 & 2814 & 1113 & 14681 \\
\hline & Porcentaje & 17.6 & 55.6 & 19.2 & 7.6 & 100 \\
\hline
\end{tabular}

Nota: Elaboración propia con datos de Rodríguez (2016).

\section{Resultados}

En términos generales, el total de los cuestionarios contestados del proyecto RPAM/SNI 2007 se distribuye de manera semejante a como se distribuía el total de los integrantes del SNI. Por supuesto, no se trata de una proporción exacta, pero bastante parecida como para tener confianza suficiente al proponer relaciones empíricas vinculadas con base en esta información.

Es importante explicar por qué dentro del área 3 los datos de RPAM/SNI 2007 registran una diferencia de tres puntos, la mayor respecto al número de investigadores del SNI en el 2007. Esto se debe a que una variable de control en este estudio fue el año de obtención del doctorado, lo que permitió realizar contrastes en las trayectorias académicas de los investigadores; sin embargo, y como señalan los criterios del SNI para esa área del conocimiento (Conacyt, 2018), no es necesario el grado de doctor, es suficiente ser médico especialista con maestría. Por tal motivo y debido al criterio de la propia institución, la representación de investigadores con doctorado es menor al número de investigadores dentro de esta área.

Por otro lado, respecto de las diferencias proporcionales de los integrantes del SNI por niveles, el conjunto de RPAM/SNI 2007 tiene una sobrerrepresentación de candidatos y una diferencia que oscila entre 2 y 3 puntos porcentuales en los restantes niveles. Al parecer los candidatos son más proclives a participar en 
este tipo de investigaciones.

Las diferencias entre la muestra y el total, por área de conocimiento y niveles, no son grandes, aunque deben ser tomadas en cuenta para matizar los resultados en cuanto a su generalización. Sin olvidar que no se trata de una muestra aleatoria, será empleada para ensayar -en esta primera aproximación al problema- las comparaciones que se han señalado.

La decisión de corte para tener grupos comparables que se ubicaran antes y después de la existencia y consolidación del SNI se realizó con base en la fecha de obtención de la licenciatura, empleando la mediana como el valor que separaba, en partes iguales, a los que, por facilidad en la comunicación de los resultados, se identificarán como "veteranos" y "jóvenes". ${ }^{3}$ La figura 1 da cuenta del resultado de esta delimitación, ubicando a cada uno de los conjuntos en relación con los períodos analíticos (Gil-Antón, 2012) que se emplean para comprender las condiciones de regulación de la profesión académica.

El primer grupo (Veteranos) se conformó con los investigadores que obtuvieron su licenciatura hasta 1987 (en ese valor se encuentra la mediana de la distribución), y los segundos (Jóvenes) cuando se obtuvo entre 1988 y 2003, año en que se registra la más reciente graduación de licenciatura en la muestra que se levantó entre finales de 2007 e inicios de 2008.

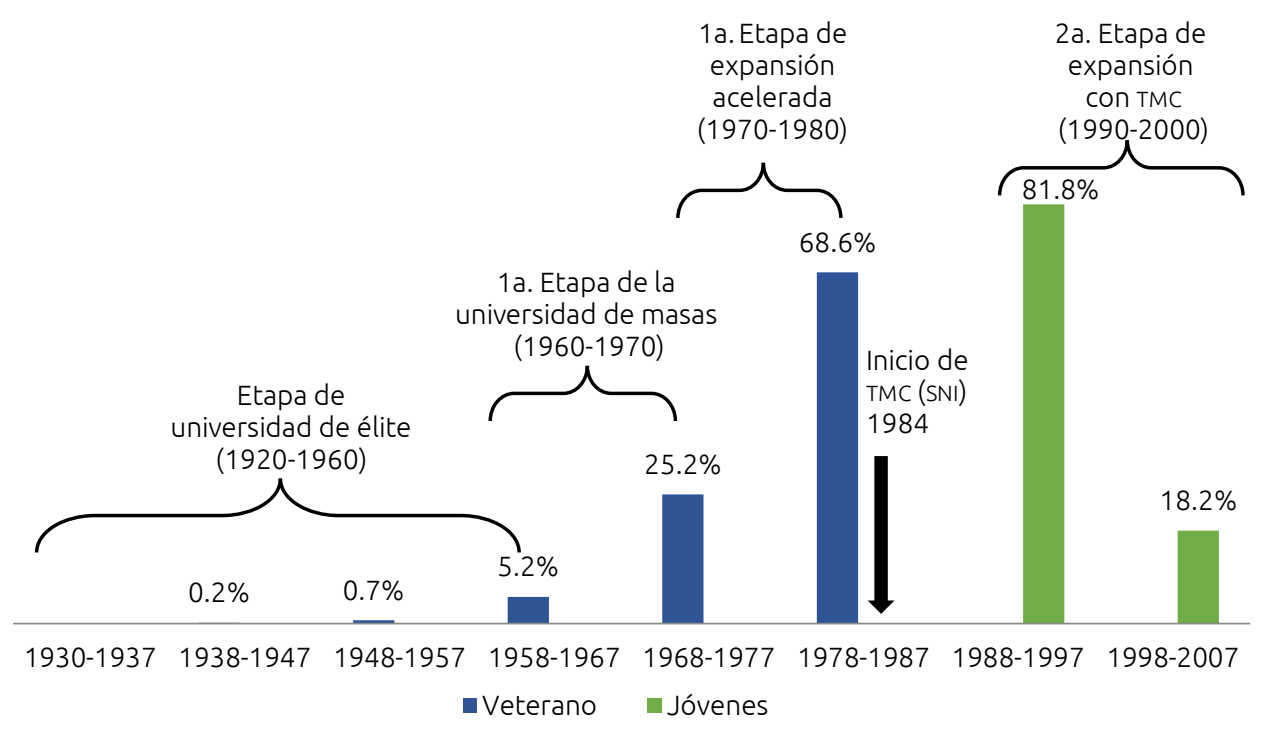

Figura 1. Distribución de la muestra RPAM/SNı por año de obtención de licenciatura

Los veteranos obtienen la licenciatura distribuidos en los períodos previos al establecimiento de las TMc: $6.1 \%$ en la etapa de la universidad de élite, previa a la expansión; $25.2 \%$ en la primera fase de la expansión hacia la universidad de masas, y el resto (68.6\%) en el período de expansión más acelerada: $50.4 \%$ del total de los que respondieron al cuestionario. Por su parte, los jóvenes (el restante $49.6 \%$ ) la consiguen ya en el contexto del establecimiento de las TMC, no sólo el SNI sino otras modalidades de pago por mérito. Son $81.8 \%$ entre 1988 y 1997 (el SNI se creó en 1984) y el resto en los años en los que "lo normal" era esta estrategia (18.2\%).

¿Se relaciona de manera significativa el momento de la obtención de la licenciatura con la edad que tienen cada uno de los conjuntos en el momento de la aplicación del cuestionario? Sería una buena aproximación a lo que se pretende comparar: grupos de veteranos y jóvenes

\footnotetext{
${ }^{3}$ El procesamiento de los datos se realizó con el programa estadístico IBM-SPSS V22.
} 
La figura 2 da cuenta de la distribución por edad del total y de cada uno de los grupos a comparar.

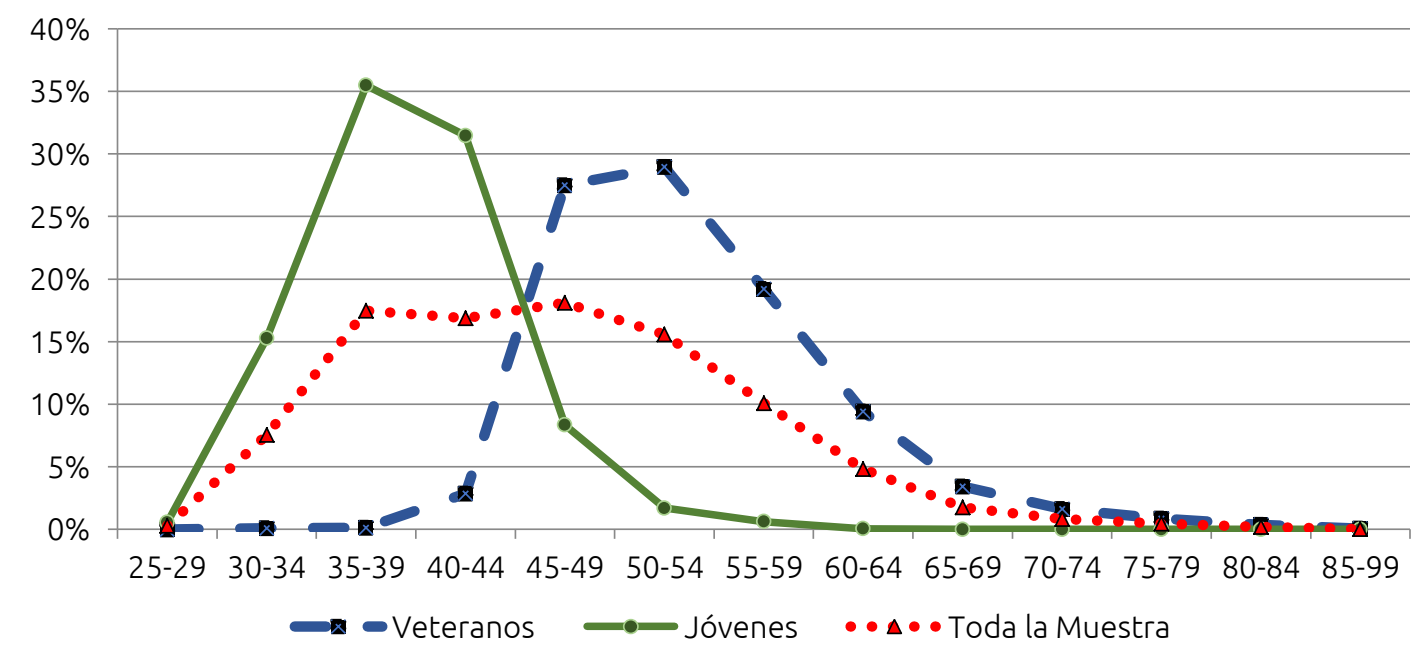

Figura 2. Edades de los investigadores de la muestra RPAM/SNI 2007

La distribución por edad de ambos grupos es congruente con la decisión de emplear la mediana en el año de obtención de la licenciatura. Los que la obtienen antes de 1988 se concentran entre los 45 y 59 años, en tanto que los que se licenciaron a partir de 1988 se agrupan entre los 30 y 44 años de edad. Con base en esta forma de distribución se realizarán las comparaciones en las secciones que siguen.

La conjetura general con la que se trabaja es:

1) Cuando se da el pasaje de la universidad de élite a la de masas en México, se presenta el fenómeno del reclutamiento acelerado de personal académico sin grados avanzados para contender con la expansión. La obtención del doctorado, por ende, llevó a este conjunto más tiempo, en concordancia con el cambio de los modos de regulación posteriores que hicieron indispensable ese grado para obtener, entre otras cosas, el ingreso al SNI y el acceso a otras modalidades de TMC. Por lo tanto, sería lógico que el grupo de los veteranos obtuviera más rápido un empleo de tiempo completo en una Institución de Educación Superior, dada la expansión, y tardase más en concluir sus estudios de doctorado, pues fue condición posterior al inicio y desarrollo de parte de su carrera en la academia.

2) En contraste, los jóvenes que ingresan a la vida académica ya con las TMC avanzando o consolidadas, van a tardar menos en graduarse como doctores, pero más tiempo en obtener un contrato de tiempo completo, debido a la saturación de las plazas por parte del conjunto de sus mayores (Gil-Antón, 2014).

En la tabla III se advierte que la conjetura tiene asidero en la información disponible sobre la edad de la obtención del doctorado y del primer contrato de tiempo completo.

Tabla III. Edades promedio de obtención de grado y años transcurridos para el primer trabajo de tiempo completo

\begin{tabular}{l|c|c|c}
\hline \multicolumn{2}{c}{ Grupos } \\
\cline { 2 - 4 } & $\begin{array}{c}\text { Todos } \\
\text { los casos }\end{array}$ & Veteranos & Jóvenes \\
\hline Edad de obtención de Licenciatura & 24.9 & 24.9 & 24.9 \\
Edad de obtención de Doctorado & 36.9 & 39.9 & 33.8 \\
Años para el primer trabajo de Tc* desde Licenciatura & 5.2 & 3.8 & 6.3 \\
\hline
\end{tabular}

$\mathrm{TC}=$ Tiempo Completo. 
Tal y como se puede ver en la tabla III, la edad promedio en que se obtiene la licenciatura es idéntica: 24.9 años para el total y ambos conjuntos. Sin embargo, la media de edad en que se doctoran es diferente: los jóvenes a los 34 años, y los veteranos a los 40 . Se cumple, pues, con el incremento en la velocidad de la formación para los primeros. Seis años menos, en promedio, es considerable e indica que, para los veteranos, el grado fue una condición no inmediata, sea, o no, impulsada por las modificaciones en la regulación del trabajo una vez que habían sido contratados. En cambio, al comparar la cantidad de años que separan a ambos grupos, en promedio, entre la licenciatura y su primer contrato de tiempo completo en una IES, pasa lo contrario: los veteranos lo consiguen en 3.8 años, mientras que los jóvenes 6.3 años después.

Mientras que la edad promedio de obtención del doctorado indica las variaciones señaladas, es posible dar cuenta también del lapso transcurrido entre la obtención de la licenciatura y la del doctorado. De ser muy diferentes y en el mismo sentido, se confirmaría la conjetura que se propone (ver figura 3).

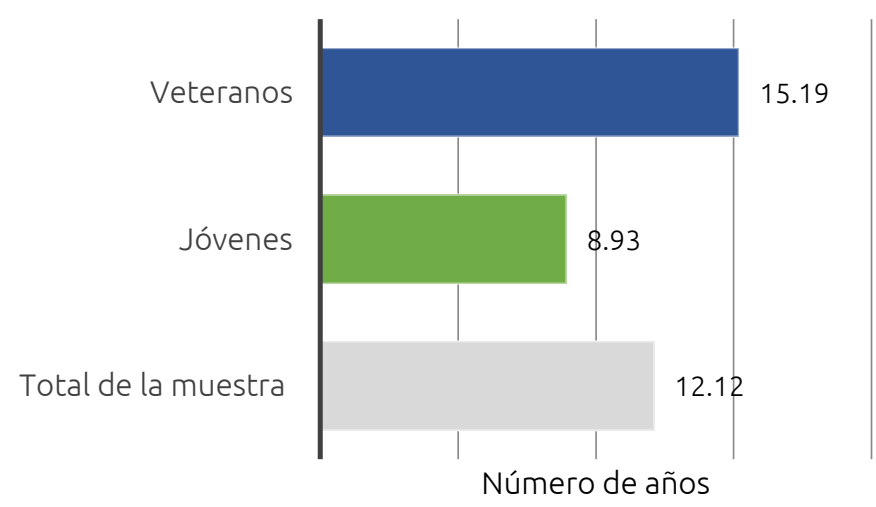

Figura 3. Número de años transcurridos desde la obtención de la licenciatura hasta la obtención del doctorado

Los años que pasan entre la obtención de la licenciatura y del doctorado son muy contrastantes. Los veteranos, en promedio, consiguen el doctorado 15 años después de licenciarse, y los jóvenes en 9 años como media.

Otra dimensión de este análisis es considerar, por quinquenios de edad y empleado el porcentaje acumulado, las edades de obtención del doctorado en ambos grupos. La figura 4 permite advertir esta variación. 


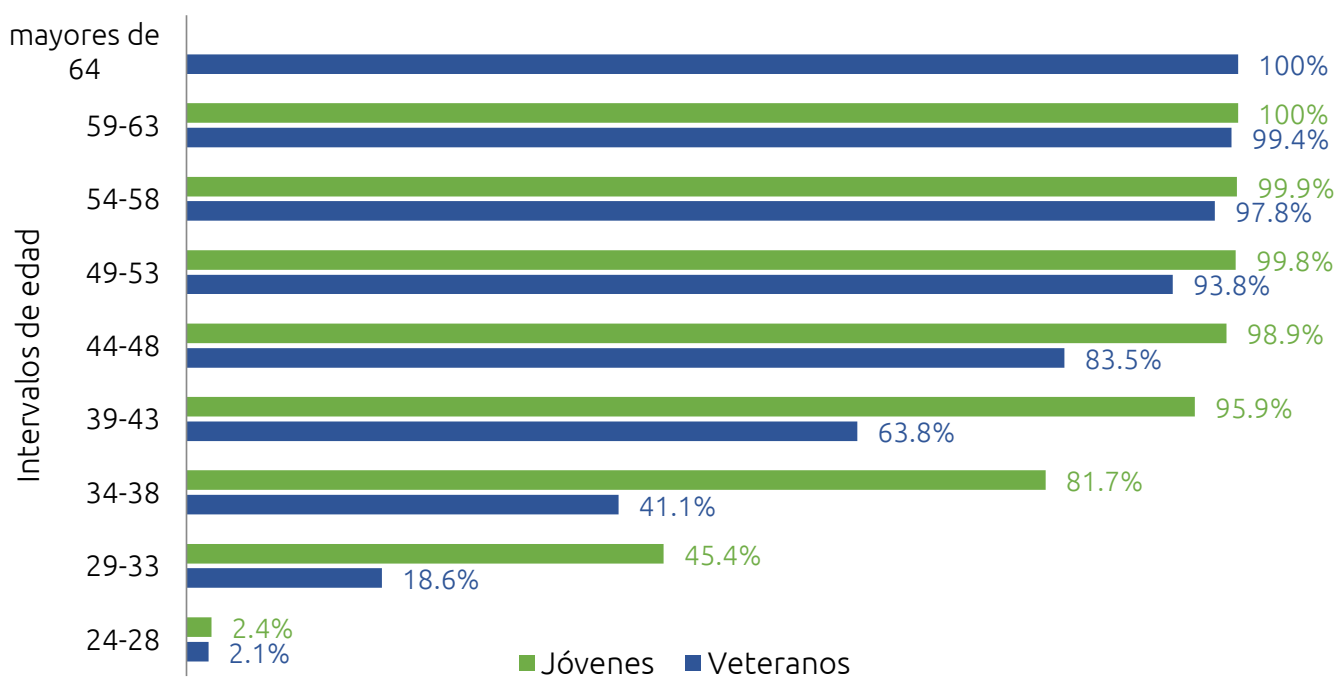

Nota: Los porcentajes que se presentan son acumulados.

Figura 4. Edad de obtención de doctorado por quinquenio

Entre los 29 y 33 años, $18.6 \%$ de los veteranos cuenta con este certificado; en contraste, casi la mitad (45.4\% de los jóvenes) obtuvo el doctorado en el mismo rango de edad. Entre los 34 y 38 años, los veteranos, como porcentaje acumulado, rebasan el $41 \%$, mientras que los jóvenes duplican la proporción (82\%). Antes de los 44 años se puede apreciar que los jóvenes han logrado este certificado en una proporción muy grande (96\%), mientras que los veteranos, para conseguir una cifra similar, lo hacen hasta el quinquenio que culmina a los 58; 11 años separan esta condición.

La velocidad a la que requieren los jóvenes doctorarse (dado el contexto de las TMC, más la escasez de puestos y el requerimiento de mayores grados para el ingreso que sus antecesores) es notablemente más rápida y obedece al cambio en las condiciones para el desarrollo de la profesión académica: por un lado, hay más académicos con mayores niveles de estudios, pero menos plazas disponibles, entre otras cosas porque los sistemas de jubilación no dan cabida a la movilidad y a la renovación de la planta.

Рara los investigadores jóvenes y los más jóvenes este fenómeno se ve reflejado en la "becarización", que consiste en pasar largos períodos posteriores a su doctorado ocupando plazas precarias (posdoctorados, estancias de investigación, becarios de investigadores, cátedras especiales para investigadores noveles) sin la certeza que en el pasado tuvo la práctica de la profesión académica, es decir, alcanzar un contrato de tiempo completo definitivo.

La tabla IV muestra el tiempo transcurrido entre la licenciatura y la obtención de un primer contrato estable y de tiempo completo, comparando los dos grupos.

Tabla IV. Número de años desde la obtención de licenciatura hasta el primer trabajo de tiempo completo

\begin{tabular}{l|c|c|c|c|c|c|c}
\hline \multicolumn{1}{c}{} & $\begin{array}{c}-10 \\
\text { antes }\end{array}$ & $\mathbf{0}$ & $\mathbf{1}$ & $\mathbf{2}$ & $\mathbf{3}$ & $\mathbf{4}$ & $\mathbf{5}$ \\
\hline Todos los casos & 7.20 & 16.30 & 22.90 & 29.70 & 36.00 & 42.60 & 49.60 \\
\hline Veteranos & 9.70 & 22.00 & 30.60 & 39.10 & 45.60 & 52.70 & 58.70 \\
\hline Jóvenes & 4.50 & 10.10 & 14.50 & 19.50 & 25.60 & 31.50 & 39.80 \\
\hline
\end{tabular}

Nota: Las cifras representan porcentajes acumulados. 
Seis años después de haber concluido la licenciatura o el número de créditos que en ese entonces se solicitaba como mínimo (-1 año), la mitad del total de la muestra había conseguido un empleo de tiempo completo. En ese mismo lapso, el 59\% los veteranos lo había logrado, mientras que los jóvenes registraron 19 puntos porcentuales menos y sólo el 40\% obtuvo un empleo. Es notable que antes de culminar la licenciatura o en el mismo año de su examen profesional, $22 \%$ de los veteranos ya contaba con empleo estable, mientras que los jóvenes, en el mismo tiempo, sólo alcanzó el 10\%.

Otra manera de presentar esta diferencia se expone en la figura 5. A los 5 años la curva es muy favorable para los veteranos en la edad temprana de obtención del tiempo completo, pero a los 9 años "empatan" y entonces, al parecer, la rápida obtención del doctorado puede hacer la diferencia para modificar la tendencia a favor de los jóvenes.

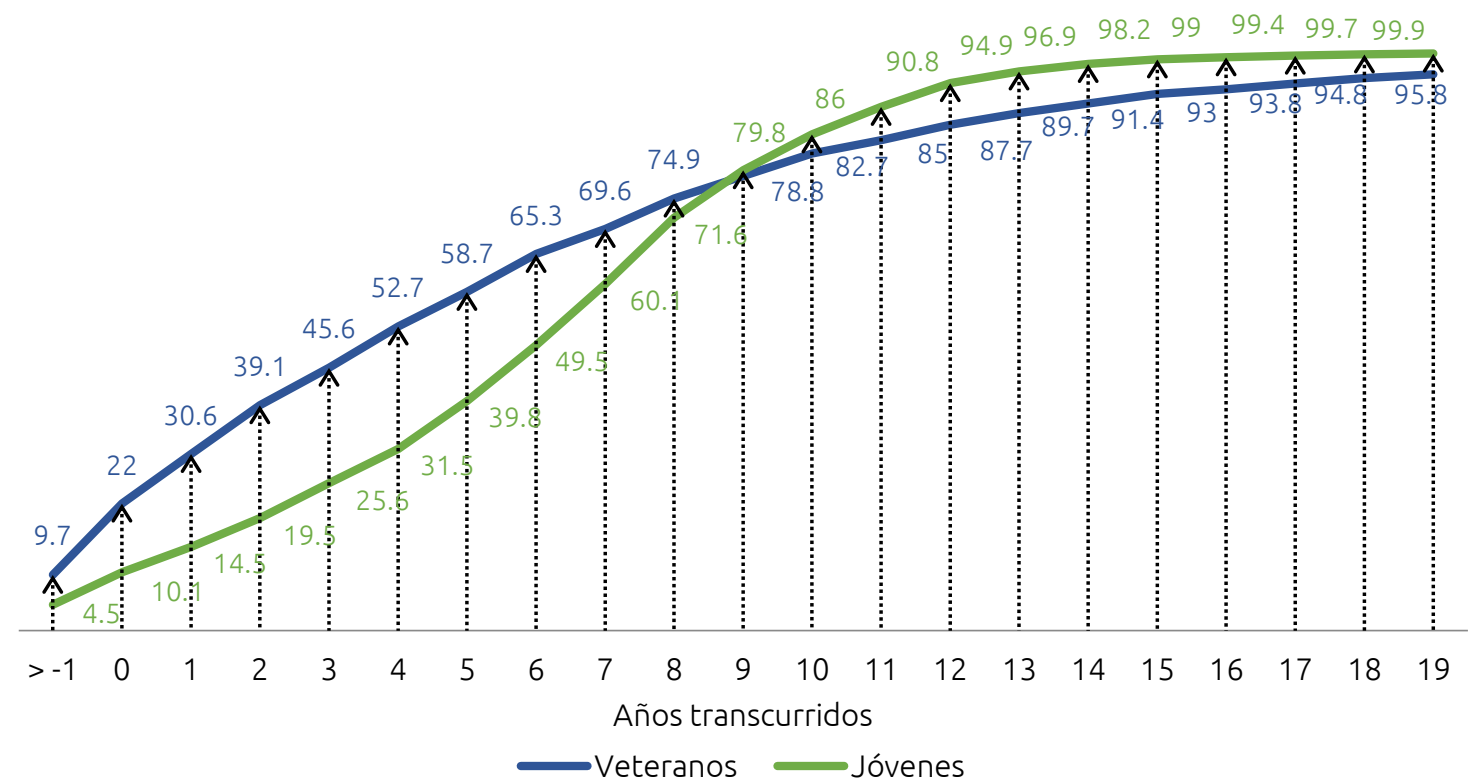

Nota: Los porcentajes que se presentan son acumulados.

Figura 5. Número de años transcurridos desde licenciatura hasta el primer trabajo de tiempo completo

Con base en los hallazgos anteriores, en efecto, las condiciones de contexto social y educativo del período salarial con expansión produjeron situaciones muy diferentes en los lapsos para obtener un puesto y para lograr el doctorado. Para los veteranos, dada la expansión y la escasa oferta de posibles académicos con posgrado, hizo que su reclutamiento laboral fuera acelerado, mientras que la tardía obtención del grado de doctor parece estar relacionada con la reingeniería derivada del establecimiento de las TMC. En contraste, los jóvenes académicos -que desarrollan sus carreras cuando el pago por mérito es una regla y los puestos académicos son ya escasos- presentan un comportamiento inverso: celeridad en la obtención del doctorado y una incorporación a la planta académica que implicó más años.

\subsection{Una mirada a las áreas del conocimiento}

La aproximación a si esto ocurrió con las mismas tendencias en diversas áreas del conocimiento es imprescindible. Gil-Antón y Contreras (2017) indican que para algunas ciencias los rasgos que premiaban las TMC ya les eran propias (espejo) y, para otras, rasgos a adquirir (modelo). 
¿Son los ethos de las distintas disciplinas tan fuertes que modificaron los diferentes ritmos en las trayectorias académicas y laborales que se han encontrado entre veteranos y jóvenes en el conjunto?

Las tradiciones disciplinarias, ¿modularon, matizaron o anularon los efectos de la variación del contexto y de las políticas derivadas de las TMC, o bien, ocurre una mezcla de circunstancia socioeducativa y tradición disciplinar?

Para acercarse a esta comparación, y con el afán de hacer un contraste más claro, juntamos el área 1 y 7 (Ciencias exactas e Ingenierías) y 4 y 5 (Humanidades y Ciencias Sociales) en la figura 6.

Los resultados son interesantes:

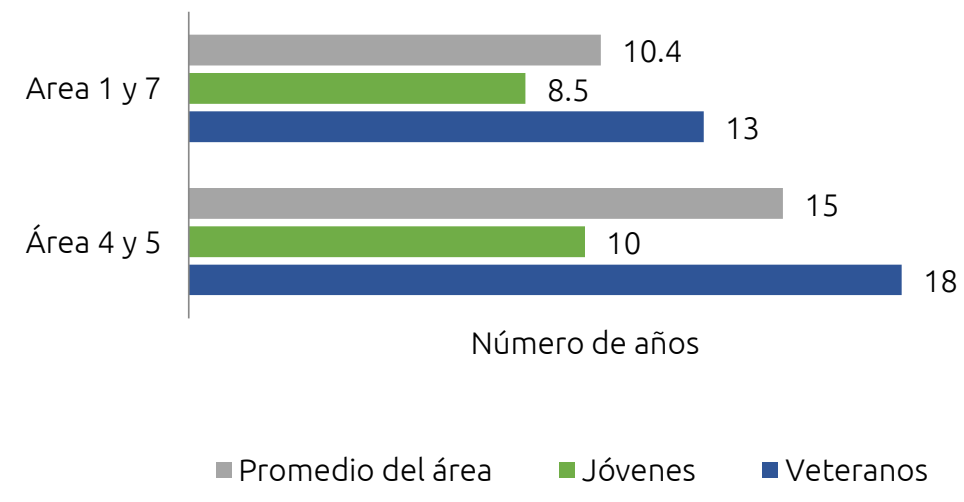

Figura 6. Número de años entre la licenciatura y el doctorado por área del conocimiento

La distancia en años entre la licenciatura y el doctorado, tanto en las áreas de las así llamadas ciencias "duras" como en las "blandas", acusan el impacto del cambio en las condiciones de contorno del sistema de regulación de la profesión académica. Como se pude ver, tanto en una como en otra, los jóvenes aceleran su trayecto formativo (en las áreas 1 y 7 bajan el promedio de los veteranos de 13 a 8.5 años, y en las 4 y 5 aún más: de 18 a 10 años).

Independiente a la tendencia general, el ethos disciplinario se manifiesta de manera clara: en el área 1 y 7 el promedio general es de 10 años entre licenciatura y doctorado; en la 4 y 5 es de 15 años, esto es, con independencia del control por grupos (veteranos y jóvenes) 5 años son una diferencia notable. Por otro lado, los veteranos del área 4 y 5 tardaron en promedio 18 años en graduarse como doctores, mientras que sus homólogos en las ciencias "exactas" lo hacen, de nuevo, 5 años antes. Pero en el caso de los jóvenes la diferencia es menor: 10 años para las Ciencias Sociales y 8.5 años para las áreas 1 y 7; es decir, hay una diferencia, sí, pero menor.

Conforme el análisis se acerca al presente, tanto las condiciones de contexto como los programas de pago por mérito y las condiciones de acceso al trabajo académico son más poderosas y tienden a reducir las variaciones entre las áreas, haciendo que las diferentes tradiciones se asemejen, por la vía de homologar la carrera académica siguiendo las modalidades propias de las ciencias "duras".

Tabla V. Promedio de edad de obtención del doctorado por áreas del conocimiento

\begin{tabular}{|c|c|c|}
\hline \multirow[b]{2}{*}{$\begin{array}{l}\text { Áreas del } \\
\text { conocimiento }\end{array}$} & \multicolumn{2}{|c|}{ Edades } \\
\hline & Veteranos & Jóvenes \\
\hline $1-7$ & 37.09 Años & 33.01 Años \\
\hline $4-5$ & 43.07 Años & 36.11 Años \\
\hline
\end{tabular}


Al observar la edad promedio en que se obtiene el doctorado (tabla V), en las áreas "duras" los jóvenes lo hacen a los 33 años, mientras los veteranos lo hicieron a los 37 (4 años de diferencia); en el caso del campo de conocimiento de las Humanidades y Sociales, de nuevo la diferencia es mucho más pronunciada: de 36 los jóvenes, a 43 los veteranos (7 años). Se constata de nuevo una tendencia en ambas áreas a reducir las edades de obtención del doctorado, mayor en las Ciencias Sociales.

No sucede lo mismo, como se puede apreciar en la tabla VI, en cuanto al tiempo que sucede entre la licenciatura y el acceso a un tiempo completo.

Tabla VI. Años desde la licenciatura al primer trabajo de tiempo completo por áreas del conocimiento

\begin{tabular}{clcccc}
\hline \multirow{2}{*}{$\begin{array}{c}\text { Áreas del } \\
\text { conocimiento }\end{array}$} & & $>-1$ & 0 & 3 & 9 \\
\hline \multirow{2}{*}{$1-7$} & Veteranos & $7.1 \%$ & $21.3 \%$ & $45.6 \%$ & $83.5 \%$ \\
& Jóvenes & $2 \%$ & $8.6 \%$ & $24.8 \%$ & $80.6 \%$ \\
\hline \multirow{2}{*}{$4-5$} & Veteranos & $11.1 \%$ & $19.7 \%$ & $45 \%$ & $73.5 \%$ \\
& Jóvenes & $7.6 \%$ & $13 \%$ & $31.1 \%$ & $77 \%$ \\
\hline
\end{tabular}

Nota: Las áreas 1-7 agrupan las ciencias exactas e ingeniería y las áreas 4-5 a las ciencias sociales y humanidades.

En este caso, los veteranos de las áreas "duras" que ingresaron antes o al año de obtener la licenciatura, así como a los 3 años, son muy semejantes a los de sus pares de las áreas "blandas", y a los 9 años la diferencia a favor de los primeros es de 10 puntos porcentuales. Entre otras razones, este comportamiento se puede explicar debido a que los veteranos de ambas áreas ocuparon las plazas disponibles en la etapa de expansión de la educación superior en México. Por ello no existe diferencia aguda entre áreas.

Sin embargo, al analizar por veteranos y jóvenes dentro de cada área sí existen diferencias, pues en las ciencias "duras" antes o el mismo año de graduación la diferencia en el porcentaje acumulado es de 12 puntos, mientras que los jóvenes de Ciencias Sociales y Humanidades presentan una diferencia de 7 puntos porcentuales para la obtención de su primer trabajo de tiempo completo.

Siguiendo el análisis de los jóvenes académicos, en específico los adscritos al área 4 y 5, su diferencia es mayor a la de sus similares del área 1 y 7 , tal vez y entre otras razones, porque en la actualidad no existe el mismo número de programas educativos a nivel nacional de las distintas áreas (Atlas de la Ciencia Mexicana, 2014; Puga y Contreras, 2016) y por lo tanto el número de plazas para estos académicos es menor.

Otro asunto a considerar es la modificación de las tendencias a los nueve años de haber obtenido la licenciatura, pues tanto los veteranos como los jóvenes de las ciencias "duras" sobrepasan las contrataciones de los académicos adscritos de las áreas "blandas". En el conjunto de los veteranos el ethos de las áreas podría ser la razón de la diferencia de 10 puntos porcentuales a favor de las "duras" (mayor tradición de obtener doctorado a edades más tempranas), mientras que los jóvenes, sólo separados por 3 puntos porcentuales, parecen guiados por la puesta en práctica de las TMc para la homologación de todas las áreas.

\subsection{Condiciones de ingreso a la profesión}

La última de las dimensiones a considerar en esta exploración es la diferencia en los niveles de estudio necesarios para la obtención del primer contrato de tiempo completo, que sintetiza y muestra el impacto de la puesta en práctica de las TMC para los jóvenes académicos, que se refleja en la tabla VII. 
Tabla VII. Grado de estudios con el que se obtuvo el primer trabajo de tiempo completo

\begin{tabular}{lll}
\hline \multirow{2}{*}{ Grado de estudios } & \multicolumn{2}{c}{ Grupos } \\
\cline { 2 - 3 } & Veteranos & Jóvenes \\
\hline TSU & $0.1 \%$ & $0.1 \%$ \\
Normal & $0.3 \%$ & $0.2 \%$ \\
Normal superior & $0.1 \%$ & $0.1 \%$ \\
\hline Licenciatura & $44.6 \%$ & $18.8 \%$ \\
\hline Especialidad & $3.1 \%$ & $0.3 \%$ \\
\hline Especialidad médica & $1.1 \%$ & $0.4 \%$ \\
\hline Maestría & $25.9 \%$ & $22.4 \%$ \\
Doctorado & $20.3 \%$ & $45.5 \%$ \\
\hline Posdoctorado & $4.5 \%$ & $12.2 \%$ \\
\hline Total & $100 \%$ & $100 \%$ \\
\hline Nota: tsu= Técnico superior universitario
\end{tabular}

El dato que muestra con mayor claridad el cambio de época entre la regulación salarial propia de la expansión y su contexto, y la puesta en marcha del pago por mérito traducido como TMc ya en otras condiciones de contorno, es que la misma proporción que antes ingresó a su primer trabajo de tiempo completo con sólo la licenciatura (44.6\%), ahora lo hace con doctorado (45.5\%).

Si la línea de corte se pone en ingresos con grados superiores a la licenciatura a partir de la maestría el contraste es, de nuevo, muy agudo. El conjunto de veteranos con estas características fue del 50.7\%, y en el caso de los jóvenes fue $80.1 \%$.

¿Cuáles son las tendencias que se entrelazaron para propiciar estos cambios? Por una parte, el contexto de abundancia de puestos de trabajo para los veteranos y posterior escasez de plazas impulsó a los jóvenes a contar con mayores credenciales para iniciar el trabajo académico. Por otra parte, la política pública en los dos períodos es muy distinta, pero decisiva, en la formación del académico y los requisitos de ingreso: en el primer caso la expansión se combinó con la ausencia relativa de personas con posgrado, lo cual hizo posible la contratación como académicos de personas con licenciatura y a veces menos. En el segundo, tanto las orientaciones de política para que todo académico cuente con doctorado como la saturación del mercado generan el crecimiento de académicos con mayor preparación al inicio de sus carreras. Incluso se puede sugerir que el 18.8 \% de los jóvenes que ingresó con sólo licenciatura lo hizo en áreas del conocimiento en que los posgrados no han adquirido carta de naturalización.

Estas consideraciones se confirman con lo que se muestra en la tabla VIII, donde se registran los grados con que ingresaron veteranos y jóvenes distinguiéndolos por área de conocimiento. Es notable cómo los veteranos de las ciencias "duras", aun en la etapa previas a las TMc y producto de su ethos disciplinar, ya ubicaron con posgrado al 60.7 \% de sus integrantes. El cálculo similar para el área de Ciencias Sociales y Humanidades es de 43.2\%. Dentro de la comparación de jóvenes de ambas áreas es nítida la influencia de las TMC, pues la diferencia se reduce considerablemente: $82.5 \%$ vs $74.1 \%$ a favor de las "duras" y una proporción de doctores sólo separada por 4 puntos porcentuales, cuando la comparación entre los veteranos es de 12 unidades. 
Tabla VIII. Grado de estudio con el que se obtuvo el primer trabajo de tiempo completo por área del conocimiento

\begin{tabular}{lcc|cc}
\hline & \multicolumn{2}{c|}{ Áreas 1 y 7 } & \multicolumn{2}{c}{ Áreas 4 y 5 } \\
\cline { 2 - 5 } Grado de estudios & Veteranos & Jóvenes & Veteranos & Jóvenes \\
\hline TSU & $0.2 \%$ & $0.1 \%$ & 0 & 0 \\
Normal & 0 & 0 & $0.8 \%$ & $1 \%$ \\
Normal Superior & 0 & 0 & 0 & $0.3 \%$ \\
\hline Licenciatura & $37.3 \%$ & $17.2 \%$ & $51.4 \%$ & $23.6 \%$ \\
Especialidad & $1.9 \%$ & $0.1 \%$ & $4.3 \%$ & $1 \%$ \\
\hline Especialidad médica & 0 & 0 & $0.3 \%$ & 0 \\
Maestría & $28.1 \%$ & $21.3 \%$ & $27.1 \%$ & $27.2 \%$ \\
\hline Doctorado & $27.3 \%$ & $48.3 \%$ & $14.9 \%$ & $43.9 \%$ \\
\hline Posdoctorado & $5.3 \%$ & $12.9 \%$ & $1.2 \%$ & $3 \%$ \\
\hline Total & $100 \%$ & $100 \%$ & $100 \%$ & $100 \%$ \\
\hline
\end{tabular}

TSU=Técnico Superior Universitario.

\section{Discusión}

La conjetura general de este artículo se basó en que, a través del tiempo y la modificación de los contextos educativos, políticos y sociales, las formas de regulación de las actividades académicas serían distintas y producirían cambios relevantes en su desarrollo.

La información disponible, con toda la prudencia que sus características implica, apoya esta perspectiva: los veteranos y los jóvenes, ya sea en conjunto o por áreas del conocimiento, transitaron en su formación y obtención de un puesto de tiempo completo de manera diferente.

Esta investigación se diferencia de otras perspectivas, pues ubica sus casos en los contextos históricos y sociales que les fueron propios y, a su vez, aborda un nivel de análisis diferente.

En primer lugar, se distancia de la perspectiva (que en ocasiones se reduce al relato de la creación del SNI) centrada en postular la importancia de su creación en el desarrollo de la ciencia y de la vida académica en México. En estos estudios no se ubica al SNI como parte de las TMC pues, desde su punto de vista, la adopción del "pago por mérito" fue una iniciativa de los científicos propuesta al gobierno mexicano, sin atender a la transformación social que implicó el fin del Estado benefactor. La ausencia de contexto y análisis de la expansión de la universidad conducen a que las explicaciones propuestas sean hagiográficas y partan del supuesto de que una política pública (o un programa) pueden prosperar por sus "méritos intrínsecos".

En relación con otros trabajos, que se toman como referentes importantes, pues analizan el surgimiento del SNI dando cuenta del contexto histórico y de transformaciones sociales relevantes como la aparición del "capitalismo académico" (Ordorika, 2004), o la adopción de mecanismos de corte productivista por parte de las élites dirigentes de la educación superior en el país (Galaz y Gil-Antón, 2009; Galaz et al., 2012; Ibarra, 2001; Ordorika y Lloyd, 2014) hay convergencia.

Por último, el trabajo se separa de una crítica general a la lógica que subyace a las TMC o al SNI, pues utiliza sus características como "hilo conductor" para dar cuenta de las transiciones entre contextos que rebasan, por ser multifactoriales, estas modalidades específicas.

\section{Conclusiones}

Con los matices y la prudencia ya señaladas, , en la transición propuesta se puede apreciar un incremento en la velocidad de la formación al máximo nivel de los jóvenes, acompañada de un plazo mayor para conseguir trabajo. El caso de los veteranos es inverso: menos "prisa" en el proceso formativo y contrataciones que implicaron menor tiempo de espera. 
Al ser -en principio- promisoria, toda veta para el estudio de la profesión académica genera una serie de cuestiones a analizar. Entre ellas se pueden señalar: las variaciones regionales, así como las propias de los distintos tipos de Instituciones de Educación Superior; la inclusión de la perspectiva de género y de las otras áreas del conocimiento que no se estudiaron y, sobre todo, ¿qué ocurrirá con el paso del tiempo y el natural retiro de los veteranos de la actividad académica, sobre todo la que tiene fuerte relación con la investigación?, ¿qué resultados se obtendrían al hacer este mismo ejercicio ahora, 10 años después de la información a la que tuvimos acceso y pudimos analizar? La agenda de investigación está abierta.

El estudio recupera una de las cuestiones más interesantes de la Sociología en general. Las modificaciones en el curso del desarrollo del oficio académico moderno -como en otras mutaciones- no son resultado de decisiones individuales "libres", sino que ocurren en los umbrales posibles que las condiciones estructurales condicionan. ¿Hay agencia -son actores- en el desarrollo de sus carreras? Por supuesto, pero esta agencia está acotada a los entornos derivados de constelaciones de variables de naturaleza socioeconómica y los tipos de política (programas y modalidades predominantes) en ambos períodos. Los académicos, entonces, no han sido ni "marionetas" apresadas por la estructura, ni actores libres de toda restricción.

\section{Referencias}

Atlas de la Ciencia Mexicana. (2014). México: Academia Mexicana de Ciencias. Recuperado de http://atlasdelacienciamexicana.org/es/index-es.shtml

Consejo Nacional de Ciencia y Tecnología. (2018). Criterios de Evaluación de la comisiones dictaminadores. Recuperado de http://conacyt.gob.mx/index.php/sni/convocatorias-conacyt/convocatorias-sistema-nacio nal-de-investigadores-sni/marco-legal-sni/criterios-sni

Diario Oficial de la Federación. (1984). Acuerdo por el cual se establece la creación del Sistema Nacional de Investigadores. Recuperado de https://www.dof.gob.mx/nota detalle.php?codigo=4680072\&fecha $=26 / 07 / 1984$

Flores, J. (2014). México llegó tarde a la Ciencia. En Reflexiones sobre ciencia, tecnología e innovación en los albores del siglo XXI (pp. 19-29). México: Gustavo Casasola-Foro Consultivo Científico y Tecnológico. (incluir el enlace de este documento)

Galaz, J. F. y Gil-Antón, M. (2009). La profesión académica en México: Un oficio en proceso de reconfiguración. Revista Electrónica de Investigación Educativa, 11(2), 1-31. Recuperado de https://redie.uabc.mx/redie/article/view/237

Galaz, J. F. y Viloria, E. (2014). La carrera del académico mexicano a principios del siglo XXI: una Exploración con base en la Encuesta RPAM 2007-2008. Revista de la Educación Superior, XLIII(171), 37-65.

Galaz, J. F., Gil-Antón, M., Padilla, L., Sevilla, J., Arcos, J. y Martínez, J. (2012). La reconfiguración de la profesión académica en México. México: Universidad Autónoma de Baja California.

García, R. (Marzo,1998). Seminario interno de la sección de Metodología y Teoría de la Ciencia. México: Centro de Investigación y de Estudios Avanzados.

Gil-Antón, M. (2012). Un cuarto de siglo: ¿El fin de una etapa en la conducción de la educación superior en México? En E. F. Cano (Ed.), La perspectiva Mexicana en el siglo XXI (pp. 264-291). México: Fondo de Cultura Económica.

Gil-Antón, M. (2014). Los jóvenes académicos en el siglo XXI: de la oportunidad inesperada al retorno del privilegio. En M. Salas y O. de Oliveira (Eds.), Desafíos y Paradojas. Los jóvenes frente a las desigualdades sociales (pp. 313-361). México: Colegio de México. 
Gil-Antón, M. y Contreras-Gómez, L. E. (2017). El Sistema Nacional de Investigadores: ¿espejo y modelo? Revista de la Educación Superior, 46(184), 1-19.

Ibarra, E. (2001). La universidad en México hoy: gubernamentalidad y modernización. México: Universidad Nacional Autónoma de México.

López-Olmedo, R., Marmolejo-Leyva, R., Perez-Angon, M. A. y Zayago-Lau, E. (2017). The role of public policies in the decentralization process of Mexican science and the formation of new researchers in institutions outside the Mexico City area. Scientometrics, 112, 1343-1366.

Malo, S. (2005). Reunión El Sistema Nacional de Investigadores a 20 años de su Fundación. En Foro Consultivo Científico y Tecnológico, Una reflexión sobre el Sistema Nacional de Investigadores a 20 años de su creación (pp.37-46). México: Foro Consultivo Científico y Tecnológico.

Ordorika, I. (2004). El mercado en la academia, En I. Ordorika (Ed.), La academia en jaque. Perspectivas sobre la evaluación de la educación superior en México (pp. 35-74). México: Universidad Nacional Autónoma de México-Porrúa.

Ordorika, I. y Lloyd, M. (2014). Teorías críticas del Estado y la disputa por la educación superior en la era de la globalización. Perfiles Educativos, 36(145), 122-139. Recuperado de http://www.iisue.unam.mx/perfiles//numeros/2014/145

Payró, S., García, V. y Aquino, S. (2016). Retos de crecimiento del Sistema Nacional de Investigadores del Consejo Nacional De Ciencia y Tecnología en México. Revista Electrónica Actualidades Investigativas en Educación, 16(2), 1-24.

Puga, C. y Contreras, O. (Coords.). (2016). Informe sobre las Ciencias Sociales en México. México: Consejo Mexicano de Ciencias Sociales-Foro Consultivo Científico y Tecnológico. Recuperado de http://foroconsultivo.org.mx/libros editados/Ciencias sociales mexico COMECSO-2016.pdf

Red de Investigadores sobre Académicos. (septiembre de 2009). Cuestionario de la Reconfiguración de la Profesión Académica en México (RPAM). Simposio realizado en el X Congreso Nacional de Investigación Educativa. Veracruz, México. Recuperado de https://www.ses.unam.mx/curso2010/pdf/M4S1-RPAMCOMIEVeracruz.pdf

Rodríguez, C. (2016). El Sistema Nacional de Investigadores en números. México. Recuperado de http://www.foroconsultivo.org.mx/libros editados/SNI en numeros.pdf

Rodríguez, J., González-Brambila, C. N. y Maqueda, G. (2018). El Sistema Nacional de Investigadores en México: 21 años de producción científica en las instituciones de educación superior (1991-2011). Investigación Bibliotecológica: archivonomía, bibliotecología e información, (Especial), 187-219.

Sarukhán, J. (2005). Reunión El Sistema Nacional de Investigadores a 20 años de su Fundación. En Foro Consultivo Científico y Tecnológico. Una reflexión sobre el Sistema Nacional de Investigadores a 20 años de su creación (pp. 32-36). México: Foro Consultivo Científico y Tecnológico.

Sarukhán, J. (2014). Aportes de la Biología a la ciencia mexicana, una larga historia. En Foro Consultivo Científico y Tecnológico, Reflexiones sobre ciencia, tecnología e innovación en los albores del siglo XXI (pp. 31-41). México: Foro Consultivo Científico y Tecnológico. 